\title{
A systematic study of stellar models for C/O-rich Wolf-Rayet stars
}

\author{
N. Langer and M. Kiriakidis \\ Universitäts-Sternwarte Göttingen, Geismarlandstraße 11 \\ D-3400 Göttingen, F.R.G.
}

A grid of homogeneous stellar models for Wolf-Rayet stars in the mass range from 1 to $60 M_{\odot}$ has been computed. For each stellar mass, stars with eight different sets of chemical compositions - from pure helium stars with $(\mathrm{Y}, \mathrm{C}, \mathrm{O})=(1,0,0)$ to extreme helium poor stars with $(\mathrm{Y}, \mathrm{C}, \mathrm{O})=(0.02,0.11,0.87)$ - have been calculated in order to investigate the dependence of the stellar structure on the stellar mass and chemical composition. Modern input physics adapted to the exotic chemical composition of the Wolf-Rayet stars has been incorporated in the models, including effects of partial recombination of helium, carbon, and oxygen, and detailed opacity tables for 20 different combinations of the $(\mathrm{Y}, \mathrm{C}, \mathrm{O})$-abundances. Furthermore, we estimated the effect of the intense, partly optically thick Wolf-Rayet winds on their apparent effective temperature, using the formalism of de Loore et al. (1982, IAU-Symp. 99, 53) with the parameters: $\dot{M}=3 \cdot 10^{-5} M_{\odot} y r^{-1}, \beta=2$, and $v_{\infty}=2000 \mathrm{~km} \mathrm{~s}^{-1}$. The main results may be summarized as follows:

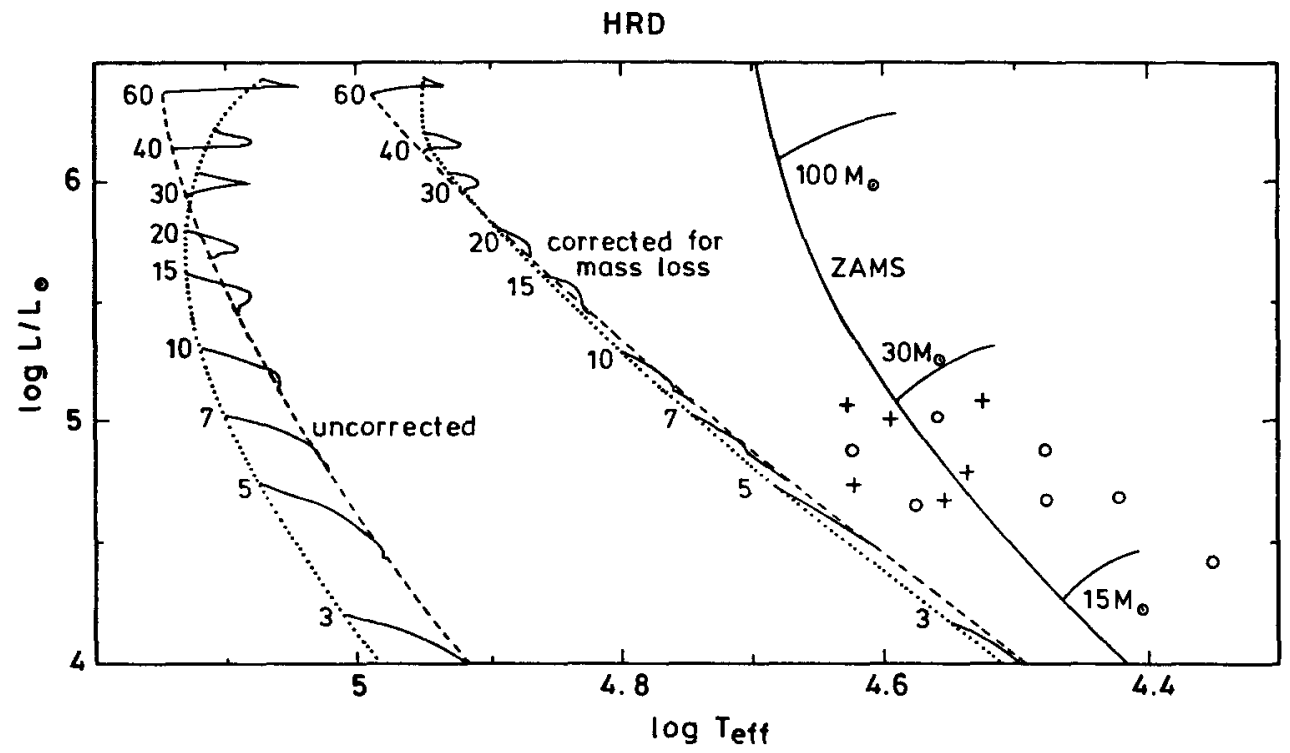

Figure 1. Lines of constant mass and varying chemical composition for the computed Wolf-Rayet models of $3,5,7,10,15,20,30,40$, and $60 M_{\odot}$ in the $H R$ diagram (solid lines). The pure helium stars are connected through a dashed line, while the extreme helium poor stars are connected through a dotted line. Also the HRD positions after applying a correction for the partly optically thick stellar wind on the effective temperature are shown. Furthermore, the theoretical zero age main sequence (ZAMS) is indicated, together with schematic evolutionary tracks for stars of 15,30 , and $100 M_{\odot}$. The crosses and circles correspond to HRD positions of observed WNE and WC stars, respectively, according to Smith and Willis (1989, Astron. Astrophys. Suppl. 54,229). 
a) Influence of the chemical composition

At high masses $\left(30-60 M_{\odot}\right)$, for a given stellar mass the models become progressively cooler and increase the radius at almost constant luminosity when the helium mass fraction decreases. This means that very massive WC stars are found to the right of their WNE counterparts (which are represented here by the pure He-stars) in the HR-diagram - at least if the stellar wind shifts the HRD positions in both cases in a similar way (cf. Fig. 1). At low mass the situation is different: For a given stellar mass, the surface temperature is increasing at approximately constant radius with decreasing helium abundance, and therefore also the luminosity is increasing.

b) Influence of the stellar mass

For low helium concentrations, the effective temperature of the WC models is increasing with decreasing mass in the range $60 M_{\odot} \geq M_{W C} \gtrsim 15 M_{\odot}$, and decreasing for $M_{W C} \lesssim 15 M_{\odot}$, while for high helium concentration the effective temperature always decreases for decreasing mass (cf. Fig. 1). However, after applying a correction to the effective temperature taking into account the stellar mass loss we find in general a strong decrease of the effective temperature with decreasing mass

The following conclusions emerge:

- At high stellar mass $\left(30 M_{\odot} \lesssim M_{W R}\right)$ the chemical composition affects the surface temperature but not the luminosity of the star. For lower masses both quantities are influenced.

- For WR stars less luminous than $\sim 3 \cdot 10^{5} L_{\odot}$ (i.e. $M_{W R} \lesssim 20 M_{\odot}$ ) no strict mass-luminosity relation exists.

- The incorporation of the effect of the intense WR mass loss on the apparent effective temperature is absolutely necessary in order to overcome the large gap between HRD positions of observed and computed WR stars.

- The stellar mass is the main parameter which determines the effective temperature of the WC stars, indicating that the so called earlier types (WCEs) correspond to higher stellar masses than the late WC stars (WCLs).

- If it is confirmed that WC stars in general have luminosities less than $\sim 10^{5} L_{\odot}$ (cf. also Schmutz et al., this volume) this would mean that WC stars have as surprisingly low masses as $M_{W C} \lesssim 10 M_{\odot}$.

Detailed results will be published in a paper now in preparation for Astronomy and Astrophysics.

N. L. gratefully acknowledges a travel grant of the Deutsche Forschungsgemeinschaft. 